\title{
THE RESPONSIVENESS OF SEXUAL OFFENDERS TO A THERAPEUTIC GROUP WORK PROGRAMME AT WESTVILLE MEDIUM B PRISON
}

\section{S Mathe}

\section{INTRODUCTION}

The scientific study of crime, has engaged the interest of many disciplines, just as professional work with offenders has drawn on the contributions of various professions, including social work. Sexual offences are among the crimes that invoke the most fear and concern. In addition to the human costs in terms of emotional and physical anguish and suffering, staggering financial costs are incurred as a result of child welfare and juvenile and criminal justice system involvement, therapeutic intervention and preventive efforts (Prentky \& Burgess, 1990).

In South Africa sexual offences are increasing at an alarming rate; this is reflected in various sources such as the media, research findings, their number in correctional services institutions, and the number of victims in therapeutic settings as well as community outcry with a range of feelings including anger, fascination, revulsion and fear. According to the statistics released by the South African Police as cited by the South African Medical Research Council (2007), 55,114 cases of rape were reported for the period 2004/2005. Since it is estimated that only one out of nine rapes is reported, the number of rapes is far larger. P4 radio news has recently reported that at the Gender Equity Conference in 2004 it was noted that in South Africa, 80 children are sexually abused every day.

Research has demonstrated that many patterns of sexual offending often begin in adolescence and, in some cases, show a progression to more serious sexual assault as adults (Grant, 2000:1). According to Linda from Childline as cited by Gerretsen (2007), more than 50\% of all sexual offences committed in South Africa and reported to Childline are committed by children under 18, with the youngest abuser being just seven years old. In February 2003, in Medium B Prison Durban Correctional Services Institution, out of 3800 sentenced offenders, 690 (30\%) were sentenced for sexual offences and 330 (close to 50\%) of them were juvenile sex offenders. Dhabicharan (2004) wrote that, the present climate in South Africa is an important factor in considering the increase in sexual offending. South Africa is a stressed and transitional community and has certain issues occurring as a result. Our country has been affected by violence during the apartheid era and the transitional period. Many South African adolescents have been exposed to violent experiences and the accompanying emotions can be traumatising. Children exposed to violence are likely to perpetrate acts of violence, and sexual offending is a sexual and violent crime. These adolescents have systematically been socialised to perceive violence and sexual control as the only viable means to assert themselves.

These statistics have called for the criminal justice system and other role players to search for a more effective means to contain the problem of sexual offending. This necessitates a joint effort of all stakeholders to ensure effective service provision in this specialized and challenging field. A common reaction to sex offenders is to seek punishment because the pain and trauma for victims are so severe. However, research data reflect that early intervention yields greater results than punishment (Blackburn, 1995). While many members of communities feel that the only safe option is continued incarceration, the reality is that most convicted sex offenders are released from custody back into their communities. 
Most corrections practitioners and researchers agree that the successful management of sex offenders lies in the combination of effective treatment and intensive, long-term supervision. Therefore, it is important that treatment programmes in corrections make a difference in the way sexual offenders think and behave and prepare them for their release and subsequent community supervision. The treatment goals should include the healing, re-education, reform and reorientation of the offender. Becker and Hunter (1997) described the main treatment objectives as preventing further victimization, halting the development of additional psychosexual problems, and helping the juvenile to develop age-appropriate relationships with peers.

Personnel in the Department of Correctional Services are faced with the challenge of rehabilitating sexual offenders placed in their care. Social workers are well placed to contribute to this rehabilitation by developing and implementing sexual offender programmes. The biggest challenge is to measure the effectiveness of Correctional Services programmes and to understand whether or not the needs of the sexual offenders are being met. King, Morris and Fitz-Gibbon (cited in Sathiparsad, 1997) stressed that the ethical and professional obligations of social workers are not simply to do their best for people, but to offer help that is most likely to be effective.

In keeping with the needs outlined above, this article presents a treatment programme developed by the researcher in consultation with the Correctional Services Treatment Task Team that was aimed towards cognitive and behaviour changes among adolescent sex offenders. Because there was no evidence of the impact of this programme on the recipients, it was difficult to gauge whether its implementation was successfully contributing to the rehabilitation of sex offenders. The researcher saw a compelling need to evaluate the programme. Feedback from the participants relating to their experiences of the programme is also discussed. Well-conceived, well-designed and thoughtfully analysed evaluation can provide valuable insights into how programmes are operating; their effectiveness, their value to programme participants, their strengths, their weaknesses, and their cost-effectiveness (Feuerstein, 1986; Herman, Morris \& Fitz-Gibbon, 1987).

\section{THEORETICAL FRAMEWORK}

\section{Cognitive-behavioural perspective}

Cognitive-behaviour modification holds that cognitions affect behaviour. Cognitive-behavioural treatment programmes draw from both empirical research on criminogenic factors associated with sex offending which identifies what treatments are likely to be relevant and effective and multimodal functional analyses of individual offenders' contemporary patterns of offending, which helps tailor treatments to the specific patterns of individual offenders (Thornton \& Hogue, 1993). Ross and Fabian (cited in Blackburn, 1995) propose that both thinking ability and content should be prime targets of rehabilitation programmes, and that the following targets are particularly relevant: social skills, interpersonal problem solving, cognitive style, social perspective taking, critical reasoning, values, meta-cognition, and self control. The general goal is to promote selfcontrol with the targets being sexual behaviour, social competence, and cognitive distortions.

There are three major classes of cognitive behaviour therapy each with a slightly different class of change goals: cognitive restructuring, coping skills therapies and problem solving therapies (Mahoney \& Arnkoff, 1978). Cognitive behavioural interventions involve cognitive analysis, thought challenging, behaviour modification, and cognitive restructuring. These are achieved through self-instructional training, thinking skills, cognitive re-education, attention-regulation and control, relaxation strategies, and skills training that are characterised by problem-solving skills, anger management skills, stress management skills, communication skills, and social skills. 
Cognitive therapy is based on the theory that distressing emotions and maladaptive behaviours are the result of faulty or irrational patterns of thinking. Dysfunctional beliefs, expectations, perceptions, attributions, interpretations and appraisals are identified and modified or replaced with rational, adaptive cognitions which alleviate the problematic feelings and behaviour (GPMHSC, 2000).

It has become evident from the literature study that a cognitive behaviour approach is probably currently the most widely used method in challenging the cognitive distortions of offenders and working to change the unacceptable behaviour. Nicolaichuk (1998) (cited in Perkins, Hammond, Coles \& Bishop, 1998:14) reports a control group follow up of 360 offenders who received group-based cognitive-behavioural treatment in Saskatoon. Nicolaichuk reports fifteen (15) year post-release experience of the treated and untreated sex offenders. A significantly smaller proportion of the treated group had sexual reconvictions of $17,6 \%$ compared with $29,9 \%$ of the control group, as well as having significantly fewer readmissions of 53,2\% as opposed to $66 \%$ of the control group. Nicolaichuk also demonstrated that the treatment group had a lower so-called "CCP slope" than the control group. The CCP slope being a measure of the seriousness of offences, which were committed as measured by number of, and sentence lengths for convictions.

The results of the study by McGrath, Cumming, Hoke and Lingston (2003) evaluating a Vermont Department of Corrections treatment programme for adult male sex offenders indicated that, men who complete cognitive-behavioural sex offender programmes sexually re-offend at relatively low rates. The number of sexual re-offenders in the completed treatment groups $(5,4 \%)$ was significantly lower than that of the some treatment $(30,6 \%)$ and no treatment groups $(30 \%)$ (McGrath et al., 2003). A review of North American sexual offender programmes revealed that most were either cognitive-behavioural or any one form or another of multi-modal or eclectic psychotherapy (Brzecki \& Wormith, cited in Marshall, 1999). Marshall (1990) further said he prefers to focus on cognitive-behavioural programmes because they appear to represent the future of sexual offenders treatment, but mostly because they are the programmes most devoted to scientific evaluations. The content of such programmes is refined by research and is responsive to scientific scrutiny (Marshall, 1990). This dependence on empirical data to guide the development of programmes is the hallmark of cognitive behavioural therapy, and it provides assurances that improvements will continue.

The conclusions drawn by Perkins et al. (1998) in their review of sex offender treatment programmes were that the treatment programmes, which utilise cognitive-behavioural treatment to address criminogenic need, can demonstrably improve survival rates.

\section{THE RESEARCH STUDY}

This study was aimed at:

- Evaluating the impact or influences that the sexual offenders treatment programme have on the sexual offenders observed and expressed beliefs, experiences, myths, thinking, perceptions, rationalization, minimization and externalisation of their behaviours, during the therapeutic group work sessions.

- Determining the strengths and weaknesses of the programme with a view to making recommendations towards the improvement of the programme.

The research took place in Westville Medium B Prison. The researcher adopted a researcherpractitioner approach. According to Strydom, Fouche and Delport (2002:59), the concept scientist-practitioner means not only that the same person can both practice and conduct research, but also that he or she can engage in practice and research simultaneously as a set of integrated 
activities. The researcher, during the study, was a social worker in Medium B Prison working with maximum security juvenile offenders. This was an evaluative research study, which adopted processes and principles of action research. These processes included need identification and assessment, consultations, literature review and formation of task teams on sexual offender treatment programme; a juvenile sexual offender treatment programme development; programme implementation; and programme evaluation. Brien (1998) asserts that action research is "learning by doing" - a group of people identifies a problem, does something to resolve it, sees how successful their efforts were, and, if not satisfied, tries again. A triangulated methodology, incorporating both qualitative and the quantitative research methods was used. The classical experimental methods represented quantitative tools, and the group process notes and observations represented qualitative methods in this study. According to Strydom et al. (2002:367), a mixed methodology design model adds complexity to a design and uses the advantages of both the qualitative and quantitative paradigms. It best mirrors the research process of working between inductive and deductive models of thinking in a research study. Marlow (1998:88) contended that combining measures can enrich one's study and can help ensure that one is tapping a maximum number of dimensions of the phenomenon under study. This method gave the researcher insight not only into the responsiveness of the research subjects to the programme, but also to the programme itself and tools that were used during the programme and the study. Purposive sampling was used for the selection of research subjects (Marlow, 1998). Only subjects that were willing to enter into the programme and who agreed to participate in a thorough assessment procedure participated in the study. Eighteen (18) sentenced juvenile offenders who committed sexual offences against female older than 12 years of age comprised the sample. Since this study tried to establish that changes in the dependant variables were the result of the introduction of the independent variable, the sample was further divided to two groups: experimental and control group. Each group comprised nine (9) research subjects. Marlow (1998) also stressed the importance of ensuring the equivalence of the comparison groups. She said equivalency of the group is important because without it we cannot determine if disparity in outcome between the two groups is due to the treatment or due to the difference between the two groups. Research subjects were randomly assigned to either the experimental group or the control group. Marlow (1998:140) recommended the use of experimental designs with randomly assigned control groups to avoid criticism such as "these are interesting findings, but it is possible that something else was influencing the results due to problems with your research design".

The primary data sources were juvenile sexual offenders. The data collection process included the pre-group phase, the group or treatment phase, and the post-group phase. The pre-group phase was characterized by opening of research subjects' case files and assessment. These files were opened by the researcher and consisted of her own notes as well as multiple sources of information such as pre-sentence reports, background reports and legal documents. Both the experimental and control groups were involved in the assessment phase. Prior to the clinical assessments, the following materials were reviewed when available: victim statement, police charge sheets, magistrates' comments, sentence warrants, prior psychological or psychiatric records, and Correctional Services admission report. According to Marshall (1999) reviewing of such documents is essential for the interviewer to challenge the offender's report. The assessment during the researcher's programme consisted of three components: a structured clinical interview, psychometric testing, and direct observations. Pre- and Post-test scales administered were Attitude Towards Women scale (Spencer \& Helmreich, 1978), Abel and Becker cognition scale (Salter, 1988) and Self-concept scale. "Measurement consists of rules for assigning numbers to 
objects so as to represent quantities or attributes numerically. This makes measurement one of the best means to create objective scientific knowledge that can enhance the professional knowledge base with the empirical evidence that is needed" (Nunnally \& Bernstein cited in Strydom et al., 2002:166).

It is important to note that even though scales are highly rated in creating objective scientific knowledge, they present a number of challenges if they are used in a cultural, linguistic and social context different to the one in which they were developed. During this study, participants with lower educational or intelligence level experienced difficulty in understanding the instructions or answering procedures of the scales. For example, they confused the relationship between the rating numbers and the value attached to them. They put 1 as an indication that they strongly agree with the statements instead of putting 4 , for instance. The researcher-practitioner ended up taking them through the whole process of answering. Since the scales are in English and the participants were Zulu speaking, the researcher practitioner spent more time trying to interpret the statement without loosing their meaning. These experiences are indicative of the need to develop scales that can be easily used by people with low education or intelligence level and that do not need language interpretation.

The group or treatment phase was characterized by the facilitation of a sexual offenders' treatment programme by the researcher. Cognitive-behavioural interventions were employed in the group programme. According to Hoghughi, Bhate and Graham (1997:130), cognitivebehavioural treatment with adolescent sexual offenders, include self-instructional training and social-cognitive skills training. Only the experimental group attended the group sessions. Research of this type raises ethical dilemmas. However, given the number of sexual offenders in prison, not all of them can be accommodated in group work sessions, even outside of a research context. Having offenders on waiting lists until they can be accommodated into the therapeutic group work programmes is not atypical. The programme was characterized by six modules with twenty three sessions. The group met on a weekly basis for approximately two hours per session. The group was also a context for data collection and provided scope for clarification and observation through consistent and repeated contacts. Different group strategies were utilized during the sessions. These strategies were group discussions, assignments, home work, audiovisual material, and each research subject's presentation. The style adopted, de-emphasized the researcher's role as teacher. Instead the role was to facilitate interactions among group members, set up discussions and sharing of experiences that would enhance members' commitment to change and deal with discouragement. All the processes of the group and the individual research subject's experiences and contributions were recorded by the researcher-practitioner.

[It is important to note that all homework given to the group members were discussed at the beginning of the group sessions.]

\section{The use of the cognitive-behavioural group as a treatment approach}

The researcher used the group work method because it is widely reported in literature as being an effective context for the treatment of sex offenders (Perkins et al., 1998). Group work reduced the risk of the researcher entering a collusive relationship with the group members, as other offenders with similar difficulties were more effective in challenging each other's thinking and behaviours. It was found that the group situation provided a safe environment for these offenders where they often, for the first time, shared openly their feelings and experiences and through the support of the other offenders provided an environment conducive for growth and change. Group work assisted in the process of change by lessening the trauma of exposure and reducing feelings of isolation. The interaction model of leadership adopted de-emphasised the researcher's role as a 
teacher. The group members, not the facilitator, were the agents of change. As a result of this leadership style, group members lowered their defences and recognised their innate strengths and abilities to change. The rich dialogue that took place during the sessions was encouraged and was beneficial to the participants. The conclusions drawn by Perkins et al. (1998) in their review of sex offender treatment programmes were that the treatment programmes, which utilise cognitivebehavioural treatment to address criminogenic need, can demonstrably improve survival rates. The cognitive-behavioural approach guided the researcher to place high regard on participants' changes in thinking, which it is hoped to contribute to change in behaviour. The programme emphases identifying thinking, experiences and knowledge that cause unwanted feelings or behaviours and to replace them with thoughts, feelings and knowledge that lead to more desirable reactions. The exploring of emotions that stemmed from the participants' beliefs, evaluations, interpretations, and reactions to life situations influenced positive change in their self-concepts, cognitions, attitude towards women, victim empathy, and their social skills. The programme under study successfully addressed the development of offenders' insights, motivation not to reoffend and the skills necessary to avoid re-offending and to achieve a non-offending lifestyle. It utilised treatment methods geared towards the needs and personal capabilities of the offenders and combinations of group therapy and individual therapy as appropriate for different aspects of treatment and assessment.

\section{Programme content}

The topics covered during group work are listed in Table 1.

TABLE 1

SEXUAL OFFENDER PROGRAMME

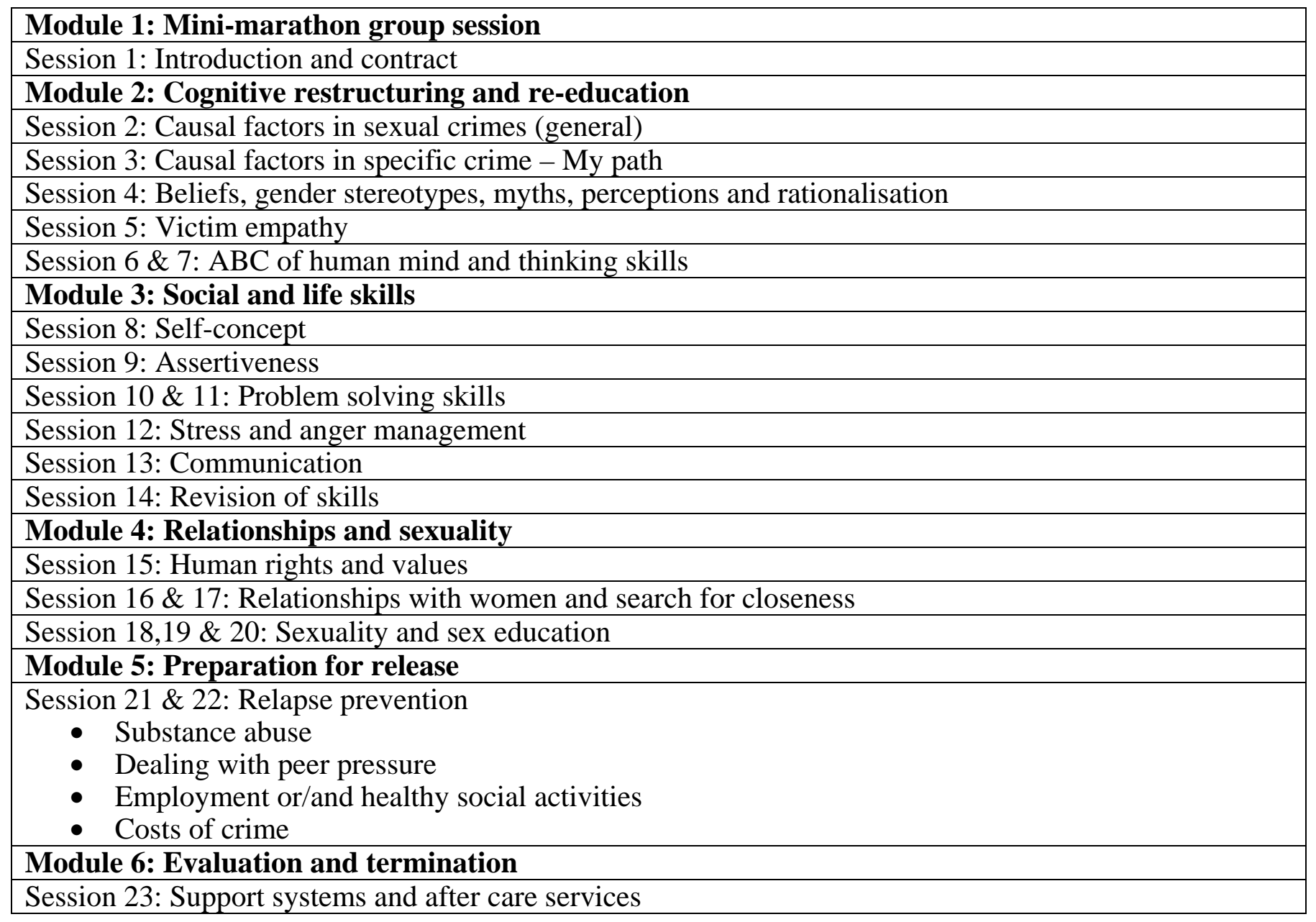




\section{Research Findings}

\section{Profiles of participants and their feedback}

The participants in this study were the four of the six types of adolescent abusers identified by Hoghughi (cited by Dhabicharan 2002:31). These are: naïve experimenter, under socialized child molester, the sexual aggressive and the group influenced offender. The causes of their offences were seen to include male dominance, female precipitation, male sexuality, socio-cultural backgrounds/socialisation, and peer pressure. Ninety percent of the participants in this study were school drop-outs with very low self -esteem; most of them were neither intellectually bright nor verbally articulate. Hoghughi et al. (1997:13) similarly found that these adolescents show particular difficulties in self-presentation, assertiveness, sympathy and sensitivity skills. Throughout the programme attention was paid to development of life, social and sexual skills. $60 \%$ of participants were observed (some of them admitted) to have poor social and sexual skills with adults and when they were unable to meet their sexual needs with adults they invariably relapse to meeting them with children or with those less powerful than them. Life, social and sexual skills learned during the group work sessions appeared to have benefited the participants. Improvement on their communication skills, self confidence, and relationship building skills was evident towards the end of the group working phase. Some participants were from traditional neighbourhoods where females are not allowed to express their views or feelings even on things concerning their lives. One participant said that in his neighbourhood, there is a common belief that women dump men that are "not men enough". Therefore to prove that one is man enough, one needs to show it through sex or through violence towards women. Similarly, in a study of 156 tribal groups, Sanday (cited in Blackburn, 1995) observed that rape prone societies were characterized by greater sex segregation, low power and status of females, and they supported ideologies favouring interpersonal violence and male toughness. One participant's offence was influenced by a traditional custom called "Ukuthwala" (taking a lady by force from her home to yours). This coincides with the feminist view that, rape is encouraged by prevailing norms of power relationships and predicts that rape will be associated with negative attitudes and beliefs about women and relations between the sexes (Blackburn, 1995). Feminist also view rape as a consequence of gender inequality, the cultural belief that women are men's property, and that rape is the outcome of patriarchal systems in which men have more power than women (Blackburn, 1995). The sessions on culture and belief systems that was aimed at talking about "things that the group members told themselves to make what they did okay or beliefs that influenced their actions", were found by participants very enlightening and helped them to develop greater insight on human rights, gender equity, and sexuality issues. All participants had adverse childhood experiences and were from families or parents with high levels of pathology including unstable family backgrounds, parental separations, violence, or experience of physical abuse or neglect. Ninety percent of them had no or little contact with their natural fathers. One participant reported that his alcoholic mother used to leave him unsupervised and without food at a very young age. He ended up being taken advantage of and being abused by relatives or neighbours whom he depended on for support. He revealed that he started to be sexually active at the age of nine (9) years of age. He reported that he was forced to have sex with his aunt who threatened to take him out of school if he reported the matter. These experiences contributed to him learning to enjoy unhealthy sex, and he held the belief that most women want to have sex with him. He said he viewed his victim (who was resisting sex) as one of those females who prefer aggressive sex since some of his own abusers wanted him to be aggressive with them when having sex. Marshall and Barbaree cited by Blackburn (1995) asserted that, as a result of adverse child experiences, such as exposure to unskilled parenting and violence, some boys fail to acquire 
appropriate empathic and social skills, which would allow them to learn this inhibition and to develop satisfying heterosexual and social interactions. Their inability to form intimate relationships also promotes aggression. Such boys are more vulnerable to cultural influences such as rape myths, and are more readily disinhibited by transitory factors, such as alcohol intoxication or anger. Participants admitted that revisiting their backgrounds and family histories was unpleasant but very beneficial. It gave them insight into their identities, their behaviours and they expressed the view that it will enable them to plan their future.

Most participants were withdrawn from society or families and were very attached to their friends, thus conforming to most of the things that friends were doing. As with delinquents in general, Becker (1998) found that sexual abusers showed a higher degree of withdrawal and social anxiety than non-sexual offenders. Three participants gang raped. Two of them were unwilling participants who conformed to the orders of a bullying friend. Most of the participants committed their offences under the influence of alcohol and they were generally using drugs and leading criminal life styles.

\section{GRAPH 1}

\section{EXPERIMENTAL GROUP SCORES: SELF-CONCEPT}

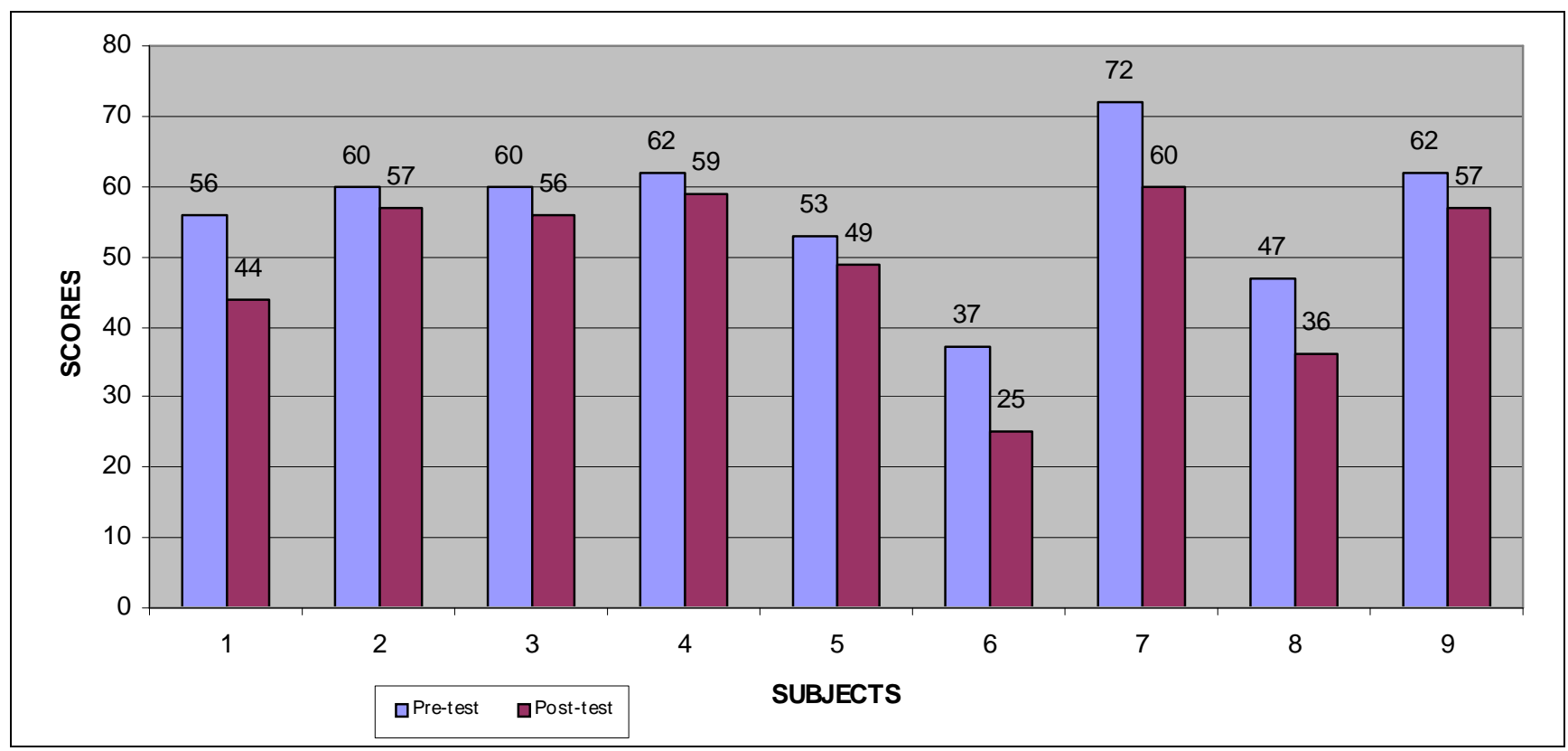

\section{The effectiveness of the sexual offender programme in helping offenders to develop a positive self-image}

The empowering sessions on self-concept, assertiveness, problem solving skills, stress and anger management, and communication skills contributed to the participants improved self-concepts. These sessions were directed towards the development of self awareness through participants' exploration of their lives; gaining or developing insight into who they were, what were their potentials; awareness about their feelings, families, and their upbringing; and learning from their own experiences, past mistakes and problem situations. According to Faller (1990) by perpetrators' understanding of their previous experiences, there can be hope of helping them to identify some of the contributing factors of abuse and the particular changes they need to make in their own relationships, thus assisting them to gain a little self-esteem. During the sessions, the group members grew from being passive participants to becoming active participants and they became self-confident. A great deal of self-disclosure occurred. The results of the pre-test and post-test are summarised in graphs one and two, followed by discussion. The Self-concept scale 
looked at the research subjects' perception of themselves and their perception of how other people view them. A score between 81-125 indicates that the subject has a quite a number of problem areas which contributes to a negative self-concept. A score between 51-80 indicates that the subject has a fair self-concept and attention should be given to the problem areas. A score between 31-50 indicates that the subject has a good self-concept with few problem areas. A score between 20-30 indicates that the subject has a very positive self-concept.

The scores presented showed high levels of improvement in self-concepts of the experimental group as compared to the control group.

It should be noted that, given the period of the programme and the fact that the group was posttested straight after the treatment, sustainability of these scores or further improvements cannot be assumed.

\section{GRAPH 2}

CONTROL GROUP SCORES: SELF-CONCEPT

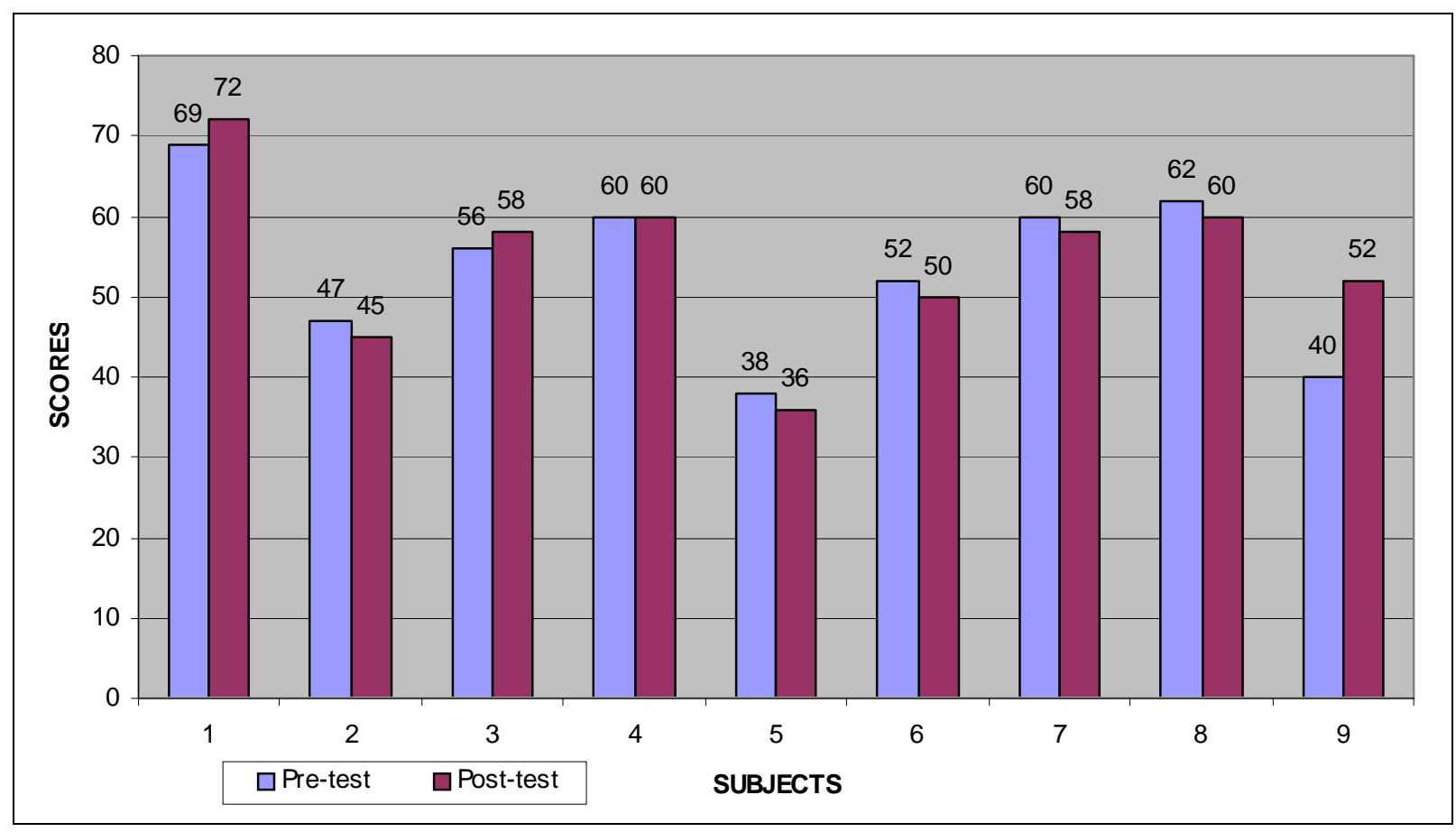

The effectiveness of the sexual offender programme in helping the offenders to develop a positive attitude towards women and to develop victim empathy

Sessions on gender issues, sexuality, values, beliefs, norms, family influences, socialization and relationships provided a platform where group members' attitudes towards women were addressed. Their responses during these sessions identified a need to take into account and address the social context within which their responses are rooted. According to Hoghughi et al. (1997), in general, we cannot understand individual behaviour in isolation from its context and the major influences on it. The group members commented on the usefulness of the role exchange exercise in developing victim empathy. Humanizing the victims prepared the way for the offenders to take responsibility for the abuse and it was only upon understanding the harmfulness of their activities, that some genuine remorse developed. The aim was to help the perpetrator to overcome the tendency to see the victim as an object for their self-expression and to see instead the victim as hurt, frightened, betrayed, and defiled by their behaviours. This module dealt, powerfully and effectively, with the implications of their behaviour for the victims. The long and short-term consequences were discussed and the focus shifted away from them to their victims, 
the victims' families and the community. This was a very powerful process and was a turning point in the treatment process. An awareness of the need to understand others' feelings and points of view before reacting to a situation was developed. The results of the pre-test and post-test of the Attitude Towards Women scale by Spencer \& Helmreich, 1978, are summarised in graphs three and four. The scale is a 15-item scale that measures attitudes towards a number of aspects of women's roles including vocational, educational and interpersonal relationships. The interpersonal realm covers dating, sexual behaviour, and marital obligations. This scale also provides information regarding offenders' degree of sex role stereotyping. Although scores range from $0-45$, it is not necessary solely to score the ATW quantitatively. The researcher also derives to information regarding the degree of subject's sex role stereotype by analysing how the subject has engaged/ answered to the scale's individual item. However a high score indicates a more egalitarian attitude toward women.

The post-test scores on the Attitude Towards Women scale administered indicated that the sexual offender programme in this study contributed to the development of a more positive attitude towards women and to greater victim empathy by the experimental group. Data obtained during the evaluation process also indicated such improvements. Group members' responses on the pretest scale, were observed to be influenced by their social backgrounds. Those from the rural areas were more prone to agree with statements that supported gender stereotypes influenced by cultural beliefs and patriarchal systems, compared with those from more urban areas.

\section{GRAPH 3}

\section{EXPERIMENTAL GROUP SCORES: ATTITUDE TOWARDS WOMEN}

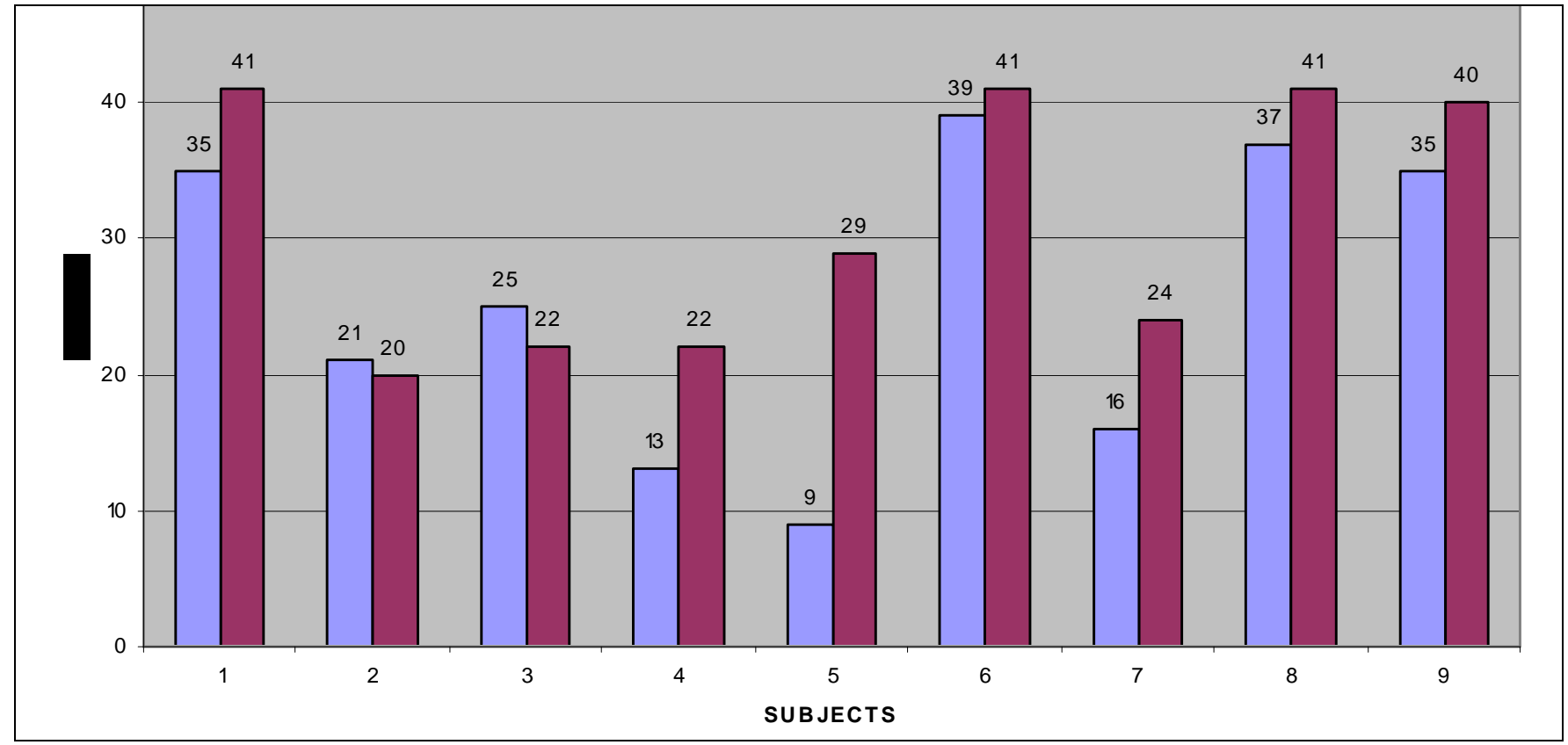




\section{GRAPH 4}

CONTROL GROUP SCORES: ATTITUDE TOWARDS WOMEN

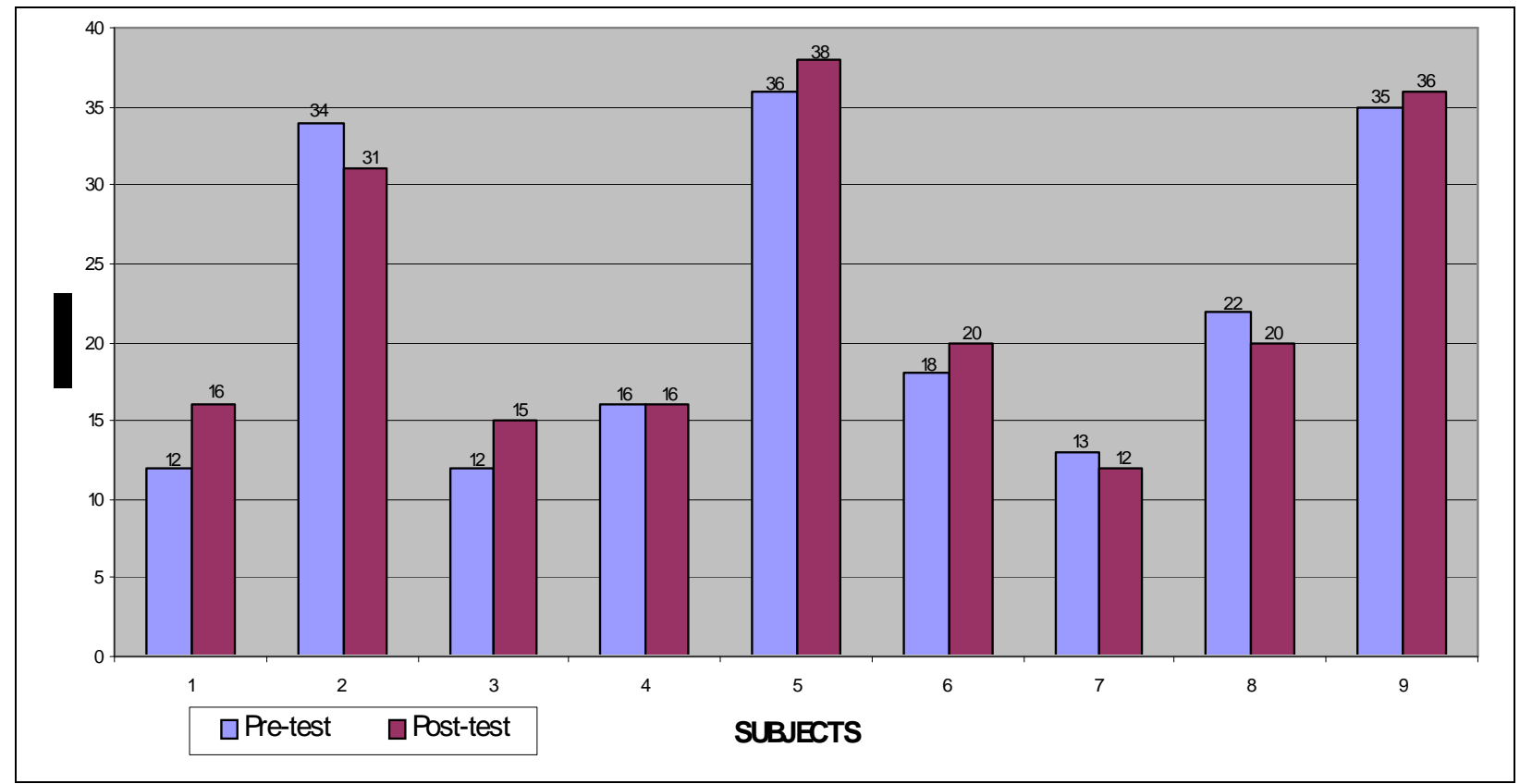

The effectiveness of the sexual offender programme in cognitive restructuring and its influence on behavioural change

Sessions on cognitive restructuring and re-education were spent developing and utilizing covert sensitisation scripts. The purpose of covert sensitisation was for each group member to learn to recognize his own thought processes and behaviours that place him at risk to abuse someone, and to interrupt these thought processes and behaviours. These sessions also provided group members with opportunities to be in touch with themselves, deal with their emotions and review and change their thinking processes. Ward, Hudson, Johnston and Marshall (1990) suggested that an understanding of the cognitive processes underlying the initiation, maintenance and justification of sexual offending is a vital pre-requisite to the successful treatment of sexual offenders. Group members' responses to these sessions indicated their increased ability to avoid taking impulsive actions or decisions. This ability can limit chances of future offending since it is strongly believed that criminal acts frequently involve the satisfaction of immediate needs at the risk of longer-term aversive consequences: criminals are commonly assumed to be deficient in control or delay functions (Hoghughi et al., 1997). The process of engaging group members in dialogue and in thought processes also had a positive impact in their improved cognition. The results of the pretest and post-test of The Abel and Becker Cognition scale as outlined by Salter (1988) are summarised in graph five and six. This is a 29 item scale that measures cognitive distortions. The items were chosen from statements offenders have actually made in treatment (Salter, 1988). The focus is on what constitutes consent to sex, the perception of passiveness in victims as permission to continue, date rape, social roles, victim as a liar, victim enjoying it, and harmfulness of sexual offences. Subjects mark each item on a scale from strongly agree to strongly disagree (1-5). The items are noted clinically rather than formally scored (Salter, 1988). Each item represents statements that have been made by sex offenders to justify their behaviour (Salter, 1988). Agreement with any of the items represents an example of distorted cognitions to be addressed in therapy. For the purpose of this graph, items are scored quantitatively as well. The scores range from 29 (strongly agree), 58 (agree), 87 (neutral), 116 (disagree) and 145 (strongly disagree). All subjects from the experimental group showed more rational, positive, and improved cognitions in their post-test scores. 
GRAPH 5

EXPERIMENTAL GROUP SCORES: COGNITION

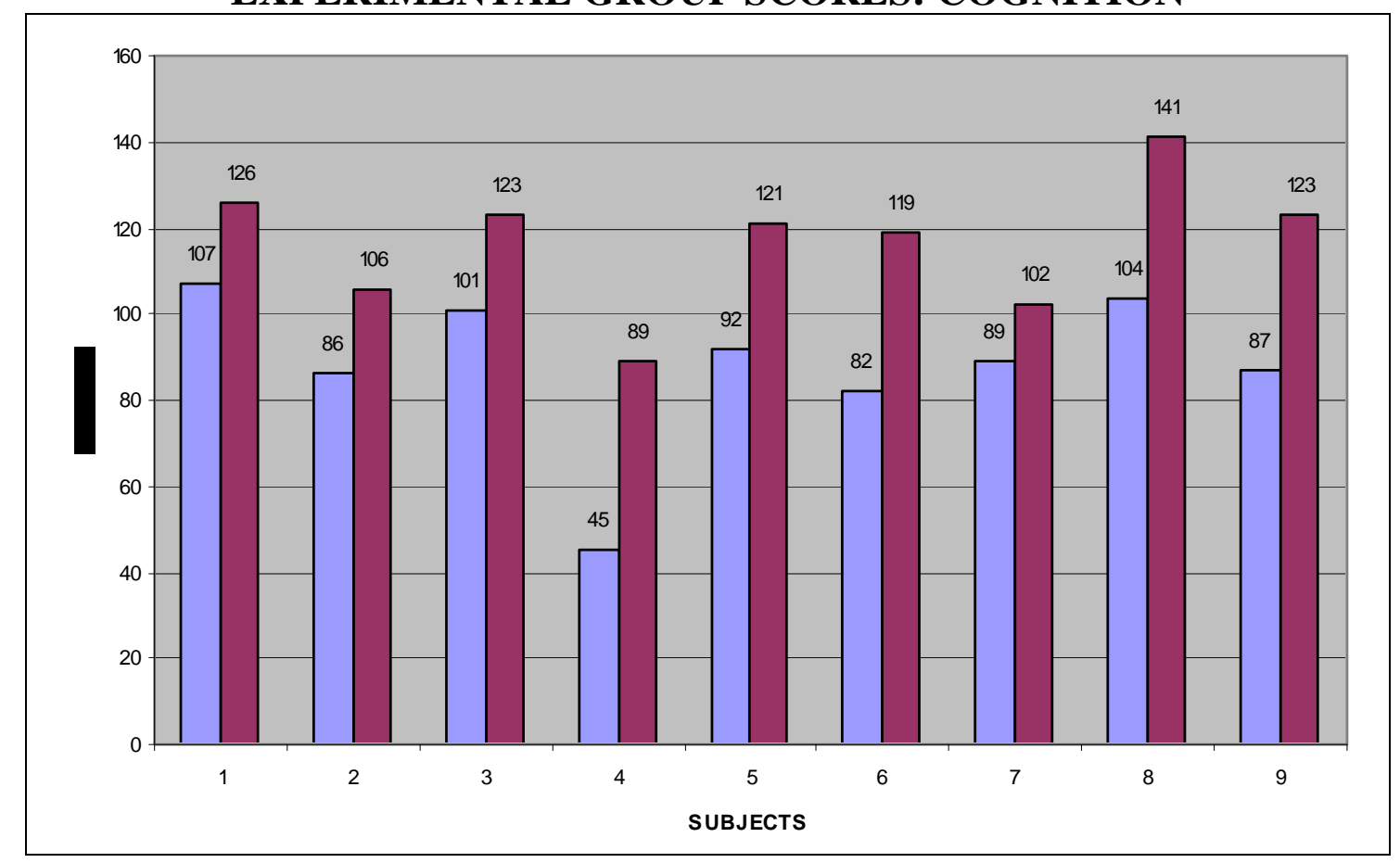

\section{GRAPH 6}

CONTROL GROUP SCORES: COGNITION

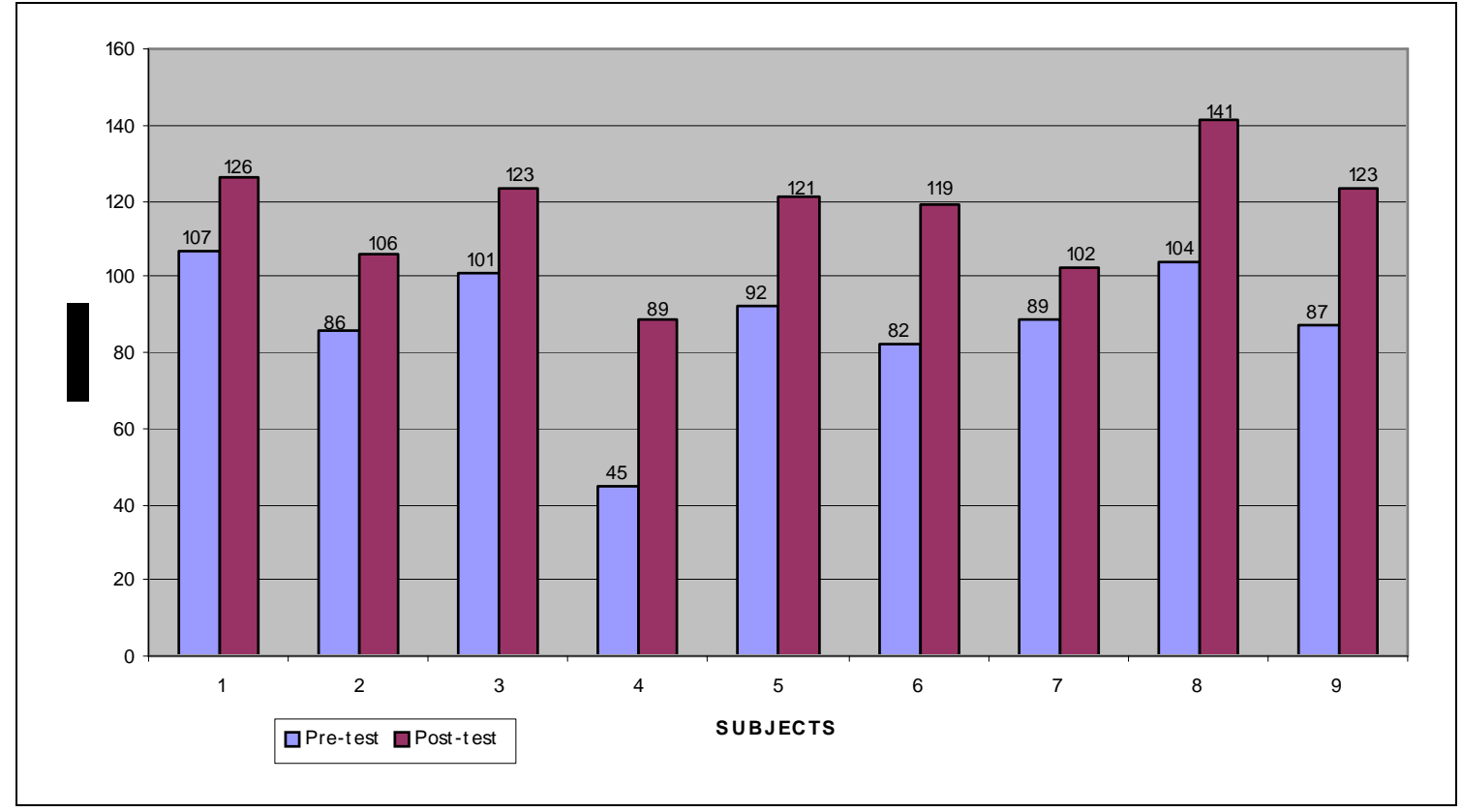

\section{FURTHER ADAPTATION OF THE PROGRAMME}

The processes of this study helped the researcher to identify factors inhibiting better programme performance and provided guidelines relating to further development and adaptation of the programme that was being evaluated. Programme adaptation may be necessary to narrow the gap between programme objectives and programme impact (Sathiparsad, 1997:109). Participants with lower educational or intelligence level experienced difficulty in understanding the instructions or answering procedures of the scales. Since the scales are in English and the participants were Zulu speaking, the researcher spent time trying to interpret the statement without loosing their 
meaning. These experiences are indicative of the need to develop scales that can be easily used by people with low education or intelligence level and that do not need language interpretation.

The need to do some exercises under supervision and during formal group sessions was identified as important in preventing future problems. For example, some participants experienced difficulty in accurately engaging in the "self-collage" exercise. Some of the participants" collages were not the reflection of the participants' lives or situations. The sessions on relationship with women and search for closeness lacked more practical components. For most participants intimate relationships were not practical while in prison. The participants were not even encouraged to develop intimate relationships with one another because that could be interpreted as promotion of homosexual relationships in prison. This aspect was identified as a need to be monitored and revisited during provision of after care services.

\section{CONCLUSION}

The overall response to the sexual offender programme in this study was positive. Data revealed that the objectives of each session and the programme as a whole were achieved. The assessment elicited information necessary for the formulation of intervention and treatment strategies, and also helped the researcher to develop a detailed profile for each research subject (Mathe, 2004). The approaches used in programme implementation were relevant and provided an environment for participants to grow and change. The topics covered in the programme were relevant and contributed to the group member's development of insight, acceptance of responsibility and accountability for their actions, motivation not to offend and the skills necessary to avoid reoffending.

The data further revealed that skills learnt during the sessions were being implemented outside of the group setting. The skills learned also inspired the group members to develop interest in improving other aspects of their lives, for example education, spirituality, and sports. However, the fact that subjects were willing participants in the programme cannot guarantee that the responses to the programme could be the same with unwilling participants. Since this was a field experiment, the researcher did not have total control of extraneous variables. Nevertheless, despite there being no proof that the change occurred solely as a result of the researcher's intervention; the triangulated research method adopted in this study, and the use of a control group supported the positive effects of the programme. This is reflected by the fact that the experimental group volunteered to continue meeting without the researcher's involvement after formal programme termination, and they became agents of change as they adopted the role of peer educators in a "no violence towards women" programme. On the other hand, given the period of the programme and the artificial environment (prison) in which the group took place; it is difficult to make comments on the sustainability of the change in the group members.

The group participants themselves during the final group evaluation expressed satisfaction about their progress and the group itself. For all of them the group experience taught them how to work in a group context, how to convey respect when interacting with people, to be patient, to confront life challenges and to make use of effective communication skills.

According to Dhabicharan (2002) there is consensus that a long-term, intensive programme has significant record of success in rehabilitating the sexual offender. Because of the unstable prison environment, it is sometimes not possible to keep a prisoner in the same prison for a long time. However, to ensure maximum effectiveness of the prison programme, it is recommended that a long term, intensive programme made up of separate interrelated modules that form the bigger rehabilitation picture be implemented. These modules could be: intensive assessment, intensive 
twenty to twenty three sessions programme similar to programme under study, restorative justice programmes including family and community re-integration, Community Corrections' after care services including relapse prevention, and other programmes such as life skills, substance abuse, and pre-release programme. These modules can be implemented separately, by different professionals, and throughout the prisoner's sentence term (from prison to parole term). Ideally, each group should have a tailor-made programme.

Community awareness programmes should also form part of the rehabilitation programmes. In the community, public acceptance of sexual stimuli and messages that foster exploitive attitudes and glorify abusive behaviours must be challenged. Social acceptance of violence, sexual objectification and compensatory sexual behaviours must be questioned. The greater success of rehabilitation programmes necessitates supportive environments by the service providers, the prison officials, prison policies, practices and procedures. These are practices that de-emphasis security over rehabilitation, policies that promote restorative justice over punishment and policies that calls for the implementation of Reconstruction and Development Programme principles. A longitudinal study is recommended to ascertain the long-term effects of the programme. In addition, studies similar to those conducted by Nicolaichuk (cited by Perkins et al., 1998) which reported fifteen year post-release experience of the treated and untreated sex offenders are recommended.

\section{BIBLIOGRAPHY}

BECKER, J.V. 1998. What we know about the characteristics and treatment of adolescents who have committed sexual offences. Child Maltreatment, 3(4):317-329.

BECKER, J.V. \& HUNTER, J.A. 1997. Understanding and treating child and adolescent sexual offenders. New York: Plenum Press.

BLACKBURN, R. 1995. The psychology of criminal conduct: Theory, research and practice. New York: John Wiley \& Sons.

BRIEN, R.O. 1998. An overview of the methodological approach of action research. Canada: University of Toronto.

DHABICHARAN, K. 2002. The life experiences of adolescent sexual offenders: Factors within the family that contribute to offending behaviours. Durban: University of Natal. London: MacMillan Press. (M thesis)

DHABICHARAN, K. 2004. Impact of domestic violence on children. Presentation at the National VEP Conference [online]. Available: Childline Kwazulu-Natal VEP Conference.htm. [Rev. 15 August 2007].

FALLER, K. 1990. Understanding and assessing child sexual maltreatment. United Kingdom: Sage Publication.

FEUERSTEIN, M.T. 1986. Partners in evaluation. London: MacMillan Press.

GERRETSEN, B. 2007. Crime and courts: Sex offence stats indict children. The Mercury, 27 April 2007:4.

GPMHSC. 2000. Detection, assessment and treatment of adolescent depression [on line]. Available: www.racgp.org.au/document. [Rev. 20 June 2003].

GRANT, A. 2000. Trends and issues in crime and criminal justice. Australia: Institute of Criminology.

HERMAN, J.L., MORRIS, L.L. \& FITZ-GIBBON, C.T. 1987. Evaluator's handbook. Newbury Park, CA: Sage Publications. 
HOGHUGHI, M.S., BHATE, S.R. \& GRAHAM, F. 1997. Working with sexually abusive adolescents. London: Sage Publications.

MAHONEY, M. \& ARNKOFF, D. 1978. Cognitive and self-control therapies. Handbook of Psychotherapy and Cognitive Behavioural Change. New York: John Wiley \& Sons.

MARLOW, C. 1998. Research methods for generalist social work. New York: Brooks/Cole Publishing Company.

MARSHALL, W. 1990. Cognitive behavioural treatment of sexual offenders. London: Chichester: Wiley.

MARSHALL, W.L. 1999. Current status of North American assessment and treatment programmes for sexual offenders. Journal of Interpersonal Violence, 14(3):221-239.

MATHE, S.Z. 2004. The responsiveness of sexual offenders to a therapeutic group work programme at Westville Medium B Prison. Durban: University of KwaZulu Natal. (M thesis)

McGRATH, R.J, CUMMING, G, HOKE, S.E \& LINGSTON, J.A. 2003. Outcome of a treatment programme for adult sex offenders from prison to community. Journal of Interpersonal Violence, 18(1):7-17.

PERKINS, D., HAMMOND, S., COLES, D. \& BISHOP, D. 1998. Review of sex offender treatment programmes [on line]. Available: http://www.ramas.co.uk/report4.pdf. [Rev. 24 April 2003].

PRENTKY, R. \& BURGESS, A.W. 1990. Rehabilitation of child molesters: A cost-benefit analysis. American Journal of Orthopsychiatry, 60(1):108-117.

SALTER, A.C. 1988. Treating child sex offenders and victims: A practical guide. Newbury Park: CA Cage.

SATHIPARSAD, R. 1997. The development and evaluation of a conflict resolution programme: The school as context. Durban: University of Natal. (M thesis)

SOUTH AFRICAN MEDICAL RESEARCH COUNCIL. 2007. Rape statistics [on line]. Available: http://www.mrc.ac.za/public/facts18.htm. [Rev. 15 August 2007].

SPENCER, J.T. \& HELMREICH, R.L. 1978. Masculinity and femininity: Their psychological dimensions, correlates and antecedents. Texas: University of Texas Press.

STRYDOM, H., FOUCHÉ, C.B. \& DELPORT, C.S.L. 2002. Evaluative research. Pretoria: South Africa.

SUSMAN, G.I. 1983. Action research: A sociotechnical systems perspective. In: MORGAN, G. (ed) Beyond method: Strategies for social research. Newbury Park, CA: Sage Publications.

THORNTON, D. \& HOGUE, T. 1993. A recent survey of sex offender treatment in the probation services. International Journal of Offender Therapy and Comparative Criminology, 40(2):375-386.

WARD, T., HUDSON, C.M., JOHNSTON, L. \& MARSHALL, W.L. 1990. Victims of sexual violence: A handbook for helpers. Singapore: Singapore University Press.

Mrs Sibonsile Mathe, Lecturer, School of Social Work \& Community Development, University of KwaZulu-Natal, Howard College, Durban, South Africa. 